schaft auf Grund der besonderen Eigenschaften des Entwicklungslandes zur persönlichen Herrschaft und zum Personenkult. In dem Umfang aber, als das Ausmaß der Loyalität gegenüber Partei, Staat und Nation von dem persönlichen Prestige des Staatschefs bestimmt wird, entscheiden seine persönlichen Geschicke das Schicksal der Institutionen. Diese Schwäche des Systems wiegt um so schwerer, als auch die Grundlagen der Partei keine Stabilität verbürgen.

Der Verfasser neigt deshalb zu der Auffassung, daß der Fortbestand des Einparteiensystems in seiner derzeitigen Gestalt befristet ist, zumal die Erfolge auf wirtschaftlichem Gebiet vielfach ausgeblieben sind oder das erwartete Ausmaß bei weitem nicht erreicht haben.

Der 3. Teil ist ganz der gegenwärtigen Gestalt und aktuellen Bedeutung der afrikanischen Einheitspartei gewidmet. Mahiou zeigt, daß die afrikanische Einheitspartei nicht den westlichen Parteien autoritären und totalitären Typs und auch nicht den kommunistischen Parteien ähnelt. Sie entzieht sich den eingeführten Instrumenten der Analyse, die nach Organisation, Funktion, sozialer Basis und Ideologie fragt. Es stellt sich heraus, daß das äußere Erscheinungsbild einer monolithischen und totalitären Partei dem wirklichen Charakter der Einheitspartei nicht entspricht. Hinter den anspruchsvollen Statuten, Texen und Verlautbarungen der Führer offenbart sich eine Organisation mit zahlreichen profunden Schwächen, die der "parti unique africain" typische Züge verleihen. Ihr Wesen aber bleibt undeutlich. Im Hintergrund zeichnet sich die Ausbildung einer führenden Gesellschaftsschicht $a b$, einer „bourgeoisie de la fonction publique", die sich zahlreicher Privilegien erfreut und in zunehmenden Gegensatz zur übrigen Gesellschaft gerät.

In den Außerungen afrikanischer Politiker erschien die Einheitspartei gelegentlich als das Wundermittel, geeignet, die Probleme Afrikas zu lösen, und auch in der wissenschaftlichen Literatur konnte es eine Zeit lang als modern gelten, das Einparteiensystem in Afrika zu preisen. Mahious Arbeit zeigt uns ein Regime, das den Erwartungen nicht entspricht. Es ist stärker, als seine praktischen Resultate vermuten lassen; aber zu schwach, um die anstehenden Aufgaben zu bewältigen. Aus dieser Sackgasse der Entwicklung kann auch das Militärregime nicht führen; der Staatsstreich allein löst jedenfalls noch nicht die Probleme, er vergrößert nur die Instabilität. Eine Zukunft verheißen dem afrikanischen Einparteienstaat die Erneuerung der Partei, der Ausbau der staatlichen Institutionen, der Entwurf einer weitschauenden Entwicklungsstrategie und, vor allem anderen, die Mobilisierung der Bevölkerung, als dem eigentlichen Träger der Entwicklung.

\section{Hartwig Rogge}

\section{GüNTER KAHLE}

Militär und Staatsbildung in den

Anfängen der Unabhängigkeit Mexikos

267 S., Lw. DM 36,-

Böhlau Verlag Köln Wien 1969

So verschieden die lateinamerikanischen Staaten im einzelnen sind, so sehr gibt es grundsätzliche Gemeinsamkeiten aus ihrer indianischen und kolonialiberischen Geschichte sowie nicht zuletzt aus den Umständen, unter denen die Staaten vor rd. 150 Jahren die Selbständigkeit erlangten. Die geistigen Grundlagen sind weitgehend für alle diese Länder gleich oder ähnlich, was vornehmlich für Spanischamerika gilt. In allen Ländern spielt auch heute noch das Militär eine ihm an sich nicht angemessene politische Rolle. In fast allen Ländern herrscht politische Unstabilität, Fortdauer der halbkolonialen Wirtschaftsstruktur und ein staatliches und verhärtetes oligarchisches Sozialgefüge, in dem immer noch der überwiegende Teil des Volkes abseits steht und an dem nominellen demokratischen Prozeß nicht teilnimmt.

Zu der Problematik der Entstehung der heutigen selbständigen Staaten aus dem 
Kolonialregime liegt jetzt bezüglich Mexiko eine Arbeit des Kölner Historikers Günter Kahle vor, die eine vorzügliche Einführung in diesen historischen $\mathrm{Ab}$ schnitt gibt. Die Arbeit vermittelt einen gründlichen Überblick über die Entwicklung des Heerwesens und sein Verhältnis zum jungen Staat Mexiko im ersten Jahrzehnt der Unabhängigkeit, d. h. in den Jahren 1821-1833. Der Verfasser geht aus von einer Schilderung des Heerwesens gegen Ende der spanischen Kolonialepoche. Die spanische Krone hat zunächst aufgrund eines sorgfältig durchdachten und ausbalancierten sowie generell auch funktionierenden zivilen Verwaltungssystems auf den Ausbau eines Heerwesens verzichten können. Erst gegen Ende der Kolonialepoche im Rahmen der bourbonischen Reformen im 18. Jahrhundert und im Zusammenhang mit der politischen Entwicklung in Europa hielt man eine systematische Aufstellung von Truppen für erforderlich. Aus den kreolischen Einwohnern wurden Miliztruppen rekrutiert, bei denen sich aber bald schon gewisse Verfallserscheinungen zeigten, nicht zuletzt gefördert durch die zu weitgehende Vergabe von individuellen Sonderrechten.

In den Unabhängigkeitskriegen erwies sich zunächst die Überlegenheit der zahlenmäßig schwachen aber regulären und disziplinierten königlichen Truppen gegenüber den improvisierten, meist aus Indios bestehenden Heeren der Aufständischen und den Guerillatruppen. Nachdem sich schließlich aber doch das Streben nach Unabhängigkeit durchgesetzt hatte, nicht zuletzt durch falsche Politik Ferdinands VII. gegenüber seinen amerikanischen Gebieten, begannen die Auseinandersetzungen um die Führungsgewalt. Die neuen Machthaber kamen aus Kreisen der Insurgenten und Guerille ros, nachdem die landbesitzende Feudal. schicht auf eine politische Führerrolle weitgehend verzichtete und sich mit dem Erhalt ihrer wirtschaftlichen Machtpositionen begnügte. Die neuen politischmilitärischen Machthaber verstanden es nicht, die staatserhaltenden Traditionen zu wahren. Die wertvollen und willigen Führungskräfte des bisherigen Regimes wurden vertrieben. Der Soldatenstand geriet in Verruf, Gewalt und Unrecht waren an der Tagesordnung. Im Vordergrund standen eigene Ansprüche der Armee, das Heer wurde zum Instrument in der Hand ehrgeiziger Offiziere, denen es um Macht und wirtschaftlichen Vorteil ging. Der Weg zu Anarchie und Diktatur war frei, wie er mindestens für die erste Hälfte des 19. Jahrhunderts typisch für ganz Spanischamerika war; die Länder kamen nicht zu einer politischen Stabilität. Das Verhältnis zwischen Staat und Heer war zerstört, das Heer war nicht mehr ein Instrument im Rahmen der Politik des Staates. Mexiko hat erst in den 30iger bis 40iger Jahren des 20. Jahrhunderts die Vorherrschaft des Heeres endgültig überwinden können, jetzt ist dem Heer die ihm zustehende Rolle in der Gesamtpolitik zugeordnet. Die Darstellung Kahles beruht auf sorgfältigem Studium der mexikanischen Quellen. Das Buch ist in allgemeinverständlichem, klaren Stil geschrieben. Die Darstellung ist von aktuellem Interesse, gerade auch um die heutigen unstabilen und oft von Militärregierungen beherrschten Verhältnissen in den spanischamerikanischen Staaten verstehen $\mathrm{zu}$ können.

Die Veröffentlichung erfolgt als Beiheft 1 zum "Jahrbuch für Geschichte von Staat, Wirtschaft und Gesellschaft Lateinamerikas". Von diesen Jahrbüchern sind bereits fünf Jahrgänge erschienen. Sie enthalten wichtigste von der deutschen historischen Lateinamerika-Forschung unter Führung ihres Seniors Richard Konetzke gesammelte Beiträge, ohne die ein Verständnis des heutigen Iberoamerika nicht mehr zu denken ist.

Friedrich Wehner 